\title{
Single-Layer Powder Coating for Furniture
}

\author{
By switching from paints to powder coatings, a furniture manufacturer not only made its coating \\ process faster, more reliable, safer for employees and more environmentally friendly, but also \\ introduced more flexibility into the design of its products.
}

The Austrian furniture company Finima found that manual painting with spray guns had distinct disadvantages when compared with powder coating. "The smells, dust and noise produced during the painting process were very unpleasant. The process also had a harmful impact on the environment. When I found that it was possible to use powder coatings, which are naturally non-toxic, my interest was immediately aroused," says Walter Schweiger, the owner of the company.

\section{Rapid coating process with low energy consumption}

The company evaluated a variety of different powder coating systems and ultimately opted for a single-layer powder coating from IGP (Rapid complete). This can be applied in one layer without a primer, which improves productivity.

As Schweiger explains: "Initially I was uncertain about the quality of this single-layer coating, but IGP helped us to identify the ideal coating thickness and the best curing parameters. It now only takes three minutes at $130^{\circ} \mathrm{C}$ to cure the coating and the results are excellent.” The new coating has a very high quality appearance. It is also water resistant and highly durable. In addition, the rapid curing process at a low temperature has allowed the company to reduce its energy costs and $\mathrm{CO}_{2}$ emissions. IGP organised a one-day technical workshop for Finima's employees to introduce them to the new coating system. They were already familiar with the principles of powder coating, but they needed to come to grips with the many parameters that influence different aspects of the coating process. For example, the employees were shown how increasing the voltage of the electrostatic field by only 10 $\mathrm{kV}$ changes the powder distribution and improves the quality of the coating. The workshop helped Finima to switch quickly and efficiently to using the one-layer coating. Since the introduction of the new coating system, IGP has worked with Finima to expand the range of colours in use and has developed new effects especially for the furniture company.

Another important reason for choosing the powder coating was the quality of the finish. "The appearance is the top priority for us," says Schweiger. "This is why we

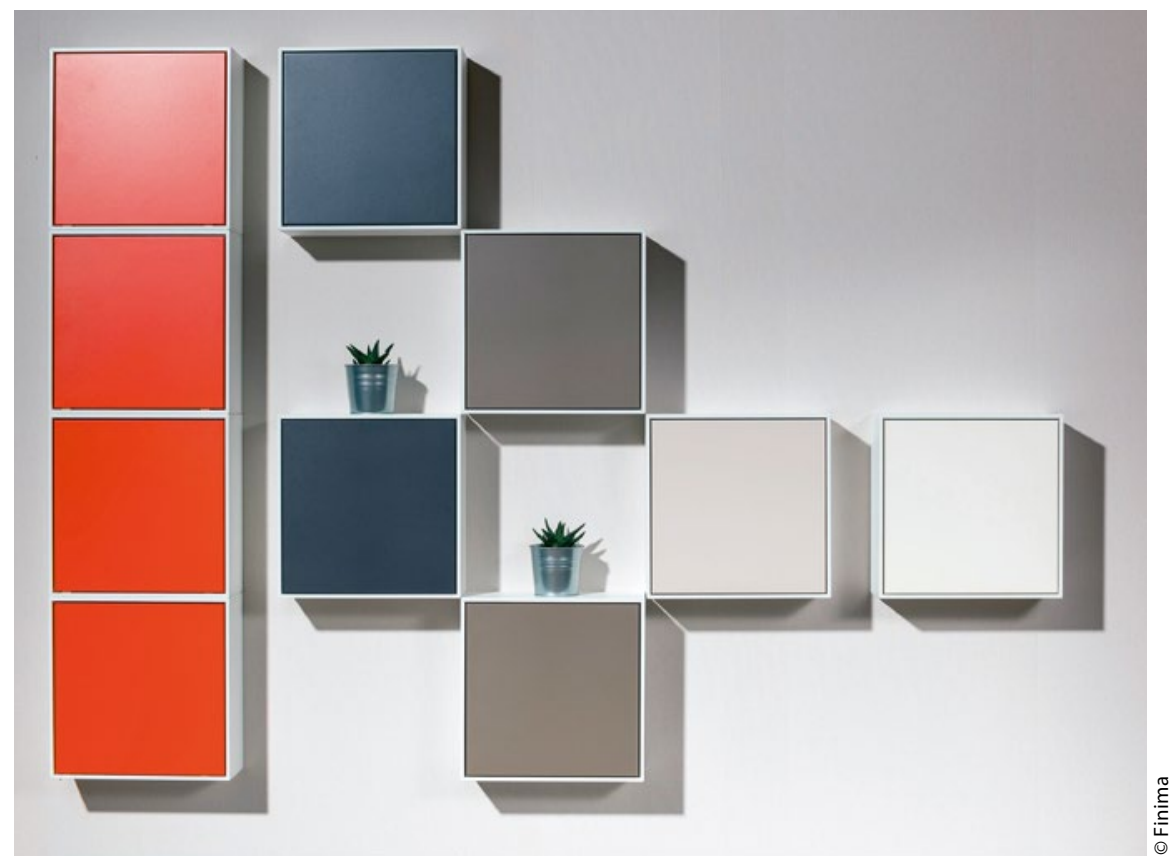

The powder coating can be applied in one layer to the wooden furniture without a primer. are very pleased that the single-layer coating looks so impressive." Powder coating also gives the company much more freedom in designing the components and the edge profiles. //

\section{Contact}

\section{IGP Pulvertechnik AG}

Wil, Switzerland

Tel. +41719298111

info@igp-powder.com

www.igp-powder.com 